\title{
Research on Automatic Fire Monitoring and Recognition Technology Based on Digital Image Processing Technology
}

\author{
Bo $\mathrm{Li}^{1,{ }^{1}}$, Tingting $\mathrm{Li}^{2}$ and Dechang Huang ${ }^{1}$ \\ ${ }^{1}$ East China Jiaotong University, Information Engineering School, Nanchang, Jiangxi, China, \\ 330013 \\ ${ }^{2}$ East China Jiaotong University, Software School, Nanchang, Jiangxi, China, 330013
}

\begin{abstract}
Keywords: Digital image processing technology; Fire monitoring; Feature extraction; Image recognition
\end{abstract}

\begin{abstract}
With the rapid development of China's industrialization and urbanization, various large-scale buildings are also emerging, and fire hazards are also increasing. Compared with the traditional fire detection technology, the visual fire detection technology based on digital image processing has the unparalleled superiority of quick response, rich information, and no constraints from large space environment, which has important social and economic value. Aiming at the main problems faced by digital image processing fire detection technology, this paper proposes an idea and method for automatic detection and recognition of flame surveillance video images. The original surveillance video image collected on-site by the visual monitoring system is the main processing object, and digital image processing technology is the main technical means. The pre-processing of surveillance video images, the segmentation of surveillance video images and the detection of suspicious flame target regions, and the analysis and extraction of feature parameters of flame image segmentation regions enable automatic detection and recognition of flame visual images.
\end{abstract}

\section{Introduction}

The losses caused by fire to human life and property are enormous. Therefore, all countries in the world attach great importance to fire monitoring and automatic fire fighting technology. So far, many scholars at home and abroad have done a lot of research on the early detection of fire, and many achievements have been made. For example, the technology of fire detection sensors has been quite mature [1-2]. The application is also very common, and the research on fire location is relatively small, mainly focused on the scanning search positioning of infrared and ultraviolet fire detectors and the location technology of fire based on GPS. For small environment, especially in indoor environment, the research of fire location technology is very few [3]. Large area spray is still the main indoor automatic fire extinguishing technology. However, because of the small scope of the fire caused by a large area of the monitoring environment of the large area of spray, often cause inestimable loss, so the fire fixed fire has become a major research direction.

With the development and popularization of computer technology, video surveillance technology and network communication technology, it is possible to detect and recognize the fire parameters in the early stages of the fire [5]. The early fire automatic detection and recognition technology of digital image processing and pattern recognition technology is used to collect the simulated video surveillance images from the fire scene by monitoring camera, and convert it into digital image by image acquisition card and input into the computer. It takes full advantage of the early fire to display visual information on the monitoring image, and combines a certain image processing and analysis algorithm to extract a variety of fire visual characteristic parameters and form a fire pattern vector in a quantitative description, and uses a pattern recognition method based on decision theory to identify and warn the fire.

\section{Preprocessing of Fire Monitoring Image}

The monitored video images collected by the monitoring system are the original RGB true color 
images. In some subsequent processing of this paper, the RGB color image needs to be converted to gray image. A gray image is a kind of monochromatic image which changes from black to white. The pixel only contains the brightness information, and does not contain color information such as hue and saturation. For the Uint 8 image usually processed by a computer, the gray value of the pixel is stored with 8 bits data, which is divided into 256 grayscale grades of $0 \sim 255$, and the gray value is valued between 0 255 [5]. The methods of color image grayscale processing mainly include maximum value method, mean value method and weighted mean value method. After a large number of experimental studies, it is known that the corresponding gray value of color pixels is obtained according to the following formula, which is more consistent with the human visual characteristics.

$$
f=0.30 \cdot R+0.59 \cdot G+0.11 \cdot B
$$

In the above formula (1), f represents the gray value corresponding to the color image pixels. In this paper, a two-dimensional function $f(x, y)$ is used to represent a frame of digital image, in which $x$ and $y$ represent the two-dimensional coordinates of the pixels, and $f(x, y)$ is the pixel point $x$, the gray value of $y$, or the color brightness value. In a computer, there are usually three representations of digital images: array representation, index image representation and two value image representation. In this paper, array representation and two value image representation are used to represent digital images. A color monitoring video image with a size of $M \times N$, is represented in a computer with a three-dimensional array of $M \times N \times 3$ (and can also be decomposed into three two-dimensional arrays of red, green and blue three color components), and then represented by a two-dimensional array of $M \times N$ size. The two value image is also represented by two dimensional array in the computer. When the two value image is only 0 (black) and 1 (white) two gray scale. In the subsequent chapters of this article, the two value image is used to represent the suspected flame image region after the segmentation, in which the pixels with a gray value of 1 represent the detected and divided suspected flame pixels.

The digital image processing toolbox (IPT) function rgb2gray in MATLAB can convert RGB color image into gray image. The syntax of function call is:

$$
I=\operatorname{rgb} 2 \operatorname{gray}(R G B)
$$

In the upper form, RGB represents true color images, and I represents converted gray level images. Figure 1 shows the true color image of RGB and the grayscale image after the conversions.

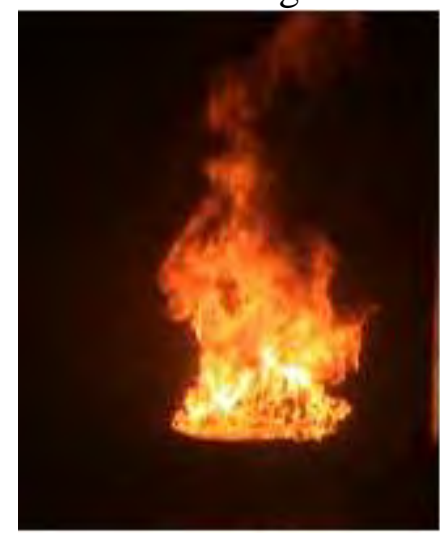

(a)RGB color image

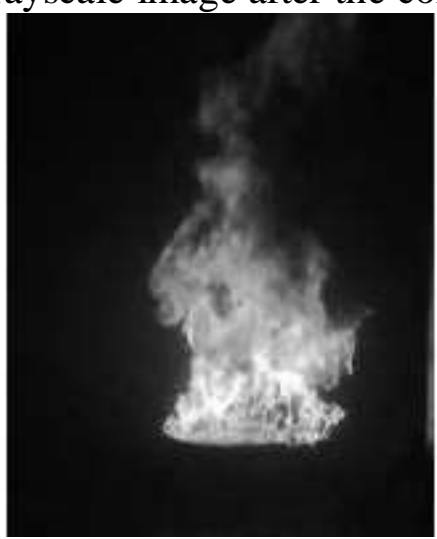

(b) Grayscale image

Figure 1. Preprocessing of flame image

Gray scale transformation is an important means of image enhancement. By increasing the dynamic range of the gray level of the image, it extends the contrast of the image so that the image is clearer and the visual features are more obvious. The gray scale transformation method is mainly divided into linear gray scale transformation, piecewise linear gray scale transformation and non-linear gray scale transformation. This paper uses the linear gray transformation processing method [6]. Assuming that the original monitoring video image, the gray value distribution of $f(x, y)$ is [a, b], $g(x, y)$ after the transformation, the Y pixel gray value range is extended to [c, d], can be realized by the following linear transformation: 
Gray scale transformation is an important means of image enhancement. By increasing the dynamic range of the gray level of the image, it extends the contrast of the image so that the image is clearer and the visual features are more obvious. The gray scale transformation method is mainly divided into linear gray scale transformation, piecewise linear gray scale transformation and nonlinear gray scale transformation. This paper uses the linear gray transformation processing method. It is assumed that the gray value distribution of the original monitoring video image $f(x, y)$ is $[a, b]$, and the $g(x, y)$ pixel gray value range of the transformed image is extended to $[c, d]$, which can be realized by the following linear transformation:

$$
g(x, y)=\frac{d-c}{b-a}(f(x, y)-a)+c
$$

\section{Histogram Modification Processing for Monitoring Video Images}

Before the histogram modification of surveillance video is processed, the concept of gray histogram is introduced. The gray histogram of the digital image is an important statistical feature of the image, which indicates the statistical relationship between each gray level in the image and the frequency of the gray level (the number of pixels with the gray level). In the gray histogram of the image, the transverse coordinates are used to represent the gradation of the pixels, and the ordinate is used to represent the frequency of the occurrence of a gray level (the normalized coordinates of the histogram represent the relative frequency, that is, the probability). The definition of grayscale histogram can be represented by the following formula.

$$
P\left(r_{k}\right)=\frac{n_{k}}{N}, k=1,2, \ldots, L
$$

In the upper form, $\mathrm{N}$ represents the total number of pixels in a frame of digital image; $\mathrm{L}$ represents the total gray level of the image; $n_{k}$ represents the frequency of the appearance of the image k gradation; $r_{k}$ represents the $\mathrm{k}$ gradation of the image, and $P\left(r_{k}\right)$ is the relative frequency of the gray level. Calling the IPT function imhist in the MATLAB image processing toolbox, you can calculate and display the gray histogram of the image, as shown in Figure 2.

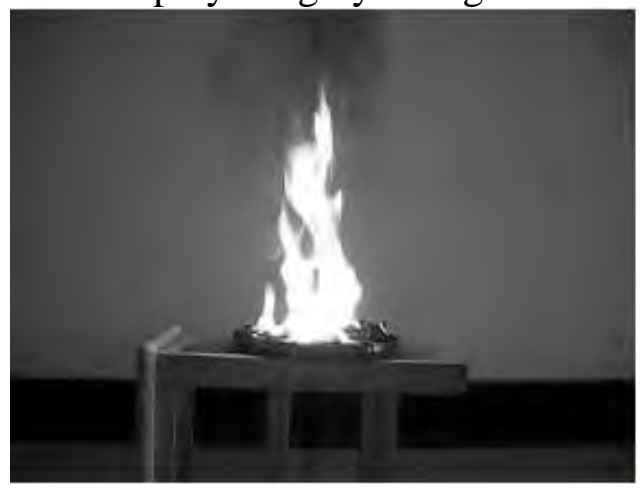

(a) Grayscale image

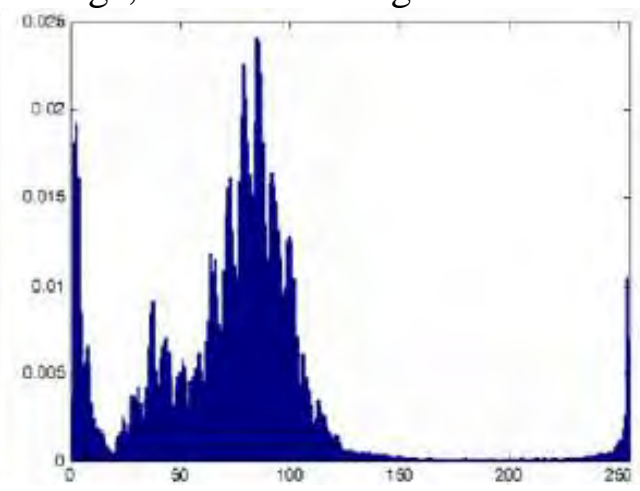

(b) Normalized histogram

Figure 2. Preprocessing of flame image

Although the gray histogram can not reflect the specific content of the image, the histogram with statistical characteristics can describe the gray distribution characteristics related to the image quality, such as the overall brightness, contrast, and the separability of objects. It is proved that by modifying the gray histogram of the image, the gray distribution of the image is opened or the gray distribution of the image tends to be balanced in the dynamic range. It can increase the variance of the image and make the details clearer, thus the purpose of image enhancement can be achieved.

The correction of grayscale histogram includes two aspects: histogram equalization and histogram normalization. In practical application, the primary difficulty of histogram normalization is that the selection of ideal histogram is difficult [7]. Therefore, the histogram equalization method is used to enhance the monitoring video image. The principle of histogram equalization is to convert 
the gray histogram of the original image through the transform function into a uniform histogram, and then the original image is trimmed according to the histogram of the equilibrium. The essence of histogram equalization is to increase the contrast between the target area and the background area by reducing the gray level of the image. Histogram equalization algorithm can be summarized as formula (2-13) and formula (2-14):

$$
\begin{gathered}
s_{k}=T\left(r_{k}\right)=\sum_{i=1}^{k} P\left(r_{k}\right)=\sum_{i=1}^{k} \frac{n_{k}}{N}, k=1,2, \ldots, L \\
r_{k}=T^{-1}\left(s_{k}\right)
\end{gathered}
$$

In the upper form, $\mathrm{T}$ represents the transformation function; $r_{k}$ and $s_{k}$ represent the original image and the gray level value of the transformed image respectively. Therefore, according to the histogram statistics of the original image, the gray level values of each image pixel after histogram equalization can be calculated. Both $r_{k}$ and $s_{k}$ are normalized and satisfied $0 \leq r_{k} \leq 1,0 \leq s_{k} \leq 1$.

\section{Description and Extraction of Dynamic Visual Characteristics of Flame Images}

The dynamic visual characteristics of the flame image are the visual features of the flame image in the digital video sequence, and the description and extraction of the dynamic visual characteristics of the flame are often the further analysis of the static visual characteristics of the flame in the time domain [8]. The development and changes of the early fire flame have the characteristics of randomness, video, hierarchy and trend. This section starts with the two aspects of the area change rate and the shape similarity of the flame area, and analyzes and extracts the dynamic visual feature parameters of the flame image by using the split flame two value image sequence.

In the initial stage of the fire incident, the flame burning has a trend of spreading growth, and the area of the flame region is constantly changing in the two value image sequence of the detected segmentation processing. The monitoring video images of 6 consecutive frames of fire flame images and other interference cases are selected. After preprocessing the front image, the split two value image region of the flame object and other interfering object regions is shown as shown in Figure 3.

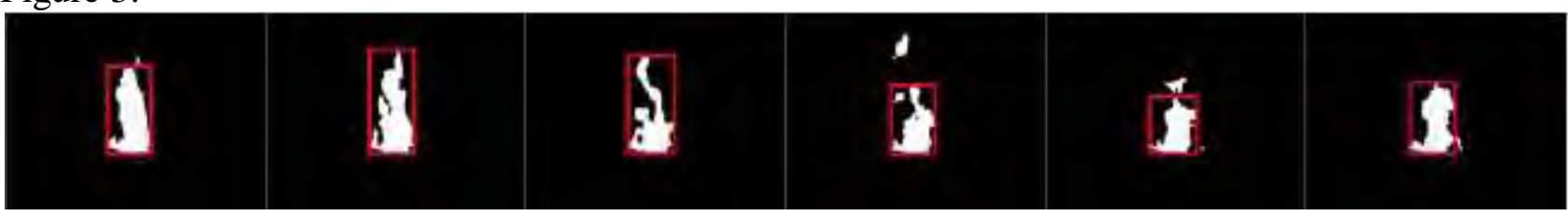

(a) Fire flame

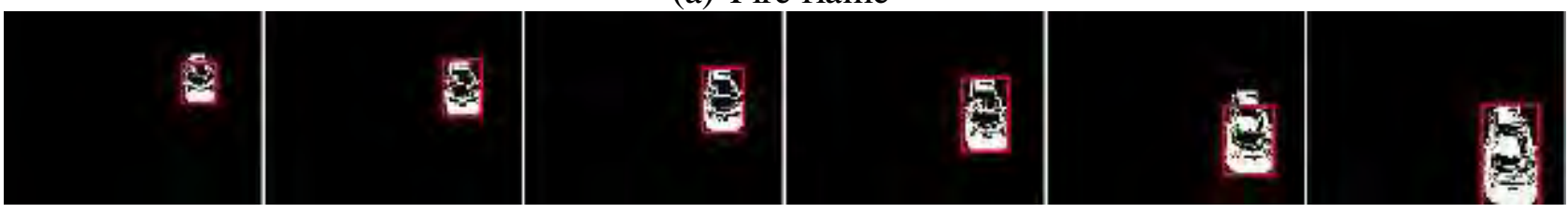

(b) Vehicle

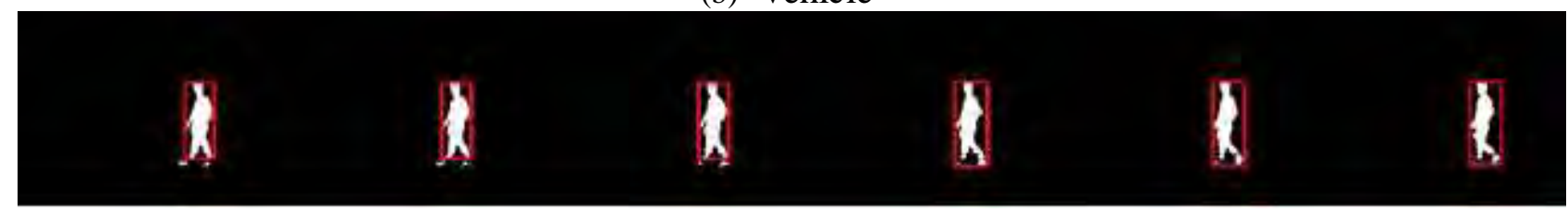

(c) Human body

Figure 3. Segmentation of fire and other interfering objects by two valued image sequences

In order to describe the dynamic visual characteristics of the flame, in order to describe the dynamic visual characteristics of the flame, this paper uses the method of calculating the relative area change rate of the two value image of the adjacent frame to characterize the area change of the flame image region. The method of calculating the area change rate of flame area is shown in formula (4-27): 


$$
G_{k}=\left|\left(A_{s k}-A_{s k-1}\right) / A_{s k}\right|, k=2,3,4, \ldots
$$

Among them, $A_{s k}$ is the area of the $S$ of the connected object area in the two value image of the frame of $k$.

\section{Hypothesis Test}

The two value images of 6 consecutive frames of flame and other interfering objects are selected to calculate the area change rate of the adjacent two frame sequence images. The calculated values are shown in Table 1.

Table 1. Characteristic values of flame area and area change rate of interfering image area

\begin{tabular}{|c|c|c|c|c|c|c|}
\hline Image sequence & 2 & 3 & 4 & 5 & 6 & Average value \\
\hline Fire flame & 0.2387 & 0.2686 & 0.2659 & 0.2139 & 0.2517 & 0.2482 \\
\hline Vehicle & 0.5539 & 0.4097 & 0.2625 & 0.3344 & 0.6799 & 0.4680 \\
\hline Human body & 0.1184 & 0.1253 & 0.1525 & 0.1579 & 0.1621 & 0.1432 \\
\hline
\end{tabular}

In the early fire flame, the area of the flame image region is constantly changing during the combustion process, and the number of pixels in the object area in the 100 frame flame two value image is calculated, as shown in Figure 4.

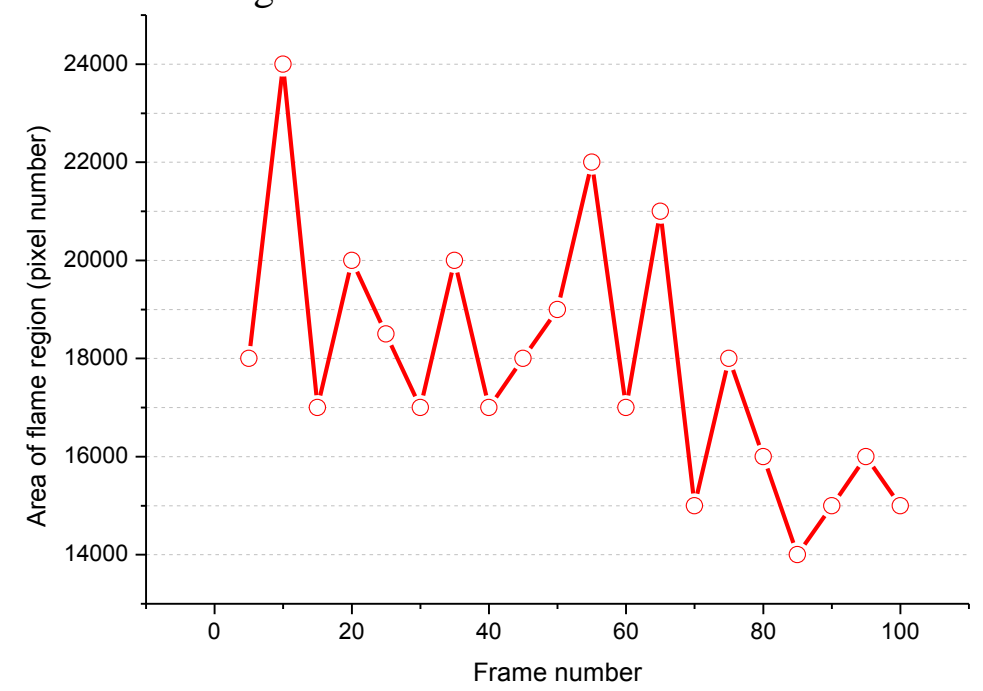

Figure 4. Area change curve of flame region

Through the analysis of the calculated value of the above area change rate, we can see that the area of the flame detection area shows a certain change law on the time axis, and the area change rate is within a certain range. For the fixed luminescent objects such as street lamps, the area change rate of the image area is obviously smaller than that in the flame area, and the area change rate of the image area is larger than the flame area for the fast moving objects such as the moving vehicles.

Based on the above analysis, the area change rate of the flame area can be used as a feature descriptor to distinguish other interference objects. But at the same time, it should be noted that the area of the object area in the image processing is obtained by the number of pixels with the pixel value of 1 in the two value image after the segmentation process. When other interfering objects move towards the camera or move from the field, the area of the suspected target area will also change, which is easy to misjudge. Because of the interference objects that may exist in the area of the flame area with similar area change rate under the actual monitoring environment, many other criteria must be integrated to identify the fire events effectively. 


\section{Conclusion}

In view of the main problems faced by the digital image processing fire detection technology, this paper proposes a method of automatic detection and recognition of the flame monitoring video image based on digital image processing technology. The original monitoring video images collected by the visual surveillance system are the main processing objects, and the digital image processing technology is the main technical means. The automatic detection and recognition of flame vision image is realized by monitoring video image preprocessing, monitoring video image segmentation, detecting suspicious flame target area, analyzing and extracting feature parameters of flame image segmentation area, and fire pattern recognition as a whole.

\section{Acknowledgment}

This work was supported by Natural Science Foundation of China (No. 61863013), Key R \& D projects of Jiangxi science and Technology Department of China (No. 20161BBE50091) and Science and Technology Foundation of Jiangxi Educational Committee of China (No. 150529).

\section{References}

[1] Fan J, Wang A, Gao Y, et al. Study on the Technology of Digital Recognition in Verification Process of Mechanical and Electronic Anemometer[J]. International Journal of Control \& Automation, 2014, 7(3):337-344.

[2] Liu X X, Huang X L, Cao L. The Design of Intellingent Fire Monitor System Based-On Digital Image[J]. Advanced Materials Research, 2012, 433-440:4178-4183.

[3] Tian Z, Wang S, University N F. Forest Fire Embedded Intelligent Monitoring System Based on BP Neural Network[J]. Journal of Northeast Forestry University, 2014, 42(8):138-653.

[4] Tian W, Song W, Ma J, et al. Experimental study of pedestrian behaviors in a corridor based on digital image processing[J]. Fire Safety Journal, 2012, 47(47):8-15.

[5] Chen J, Bao Q. Digital Image Processing based Fire Flame Color and Oscillation Frequency Analysis[J]. Procedia Engineering, 2012, 45(2):595-601.

[6] Chung Y F, Hsiao T C, Chen S C. The Application of RFID Monitoring Technology to Patrol Management System in Petrochemical Industry[J]. Wireless Personal Communications, 2014, 79(2):1063-1088.

[7] Liu X F, Geng Y, He Z M, et al. Design and Realization of Forest Fire Monitoring System Based on GIS in Henan Province, China[J]. Advanced Materials Research, 2013, 610-613:3665-3669.

[8] Burnett J D, Wing M G. A low-cost near-infrared digital camera for fire detection and monitoring[J]. International Journal of Remote Sensing, 2018, 39(3):741-753. 